\title{
Pulpectomy versus pulpotomy in the treatment primary molars with chronic pulpitis
}

\author{
Elena Brusnitsyna*, Taras Zakirov, Evgeniy Ioshenko, Maria Saipeeva, and Tatiana Stati \\ Ural State Medical University, Department of Pediatric Dentistry and Orthodontics, 620109, Repina \\ st., 3, Ekaterinburg, Russia
}

\begin{abstract}
The purpose of this study was to compare outcomes and survival of pulpectomy and pulpotomy in primary molars with chronic fibrous pulpitis. Significant differences in radiological failure rates were found among the groups of pulpectomy and pulpotomy ( $R R=2.00$, 95\% CI 1.30 to 3.07). No significant differences post-treatment survival were observed among the groups: $26.46 \pm 1.36$ months and $25.76 \pm 1.47(\mathrm{p}=0.730)$.
\end{abstract}

\section{Introduction}

The prevalence of caries complications in children is high. Voronin P.A. (2019) provides data that chronic fibrous pulpitis is most common from 1.5 to 8 years old - in $85 \%$ of cases [1]. The relevance of the study of treatment methods for chronic pulpitis is obvious. AAPD Guideline formulated treatment goals: the root pulp should remain asymptomatic, without adverse clinical signs or symptoms, such as sensitivity, pain, or swelling. There should be no harm to the germ of a permanent tooth [2]. The diagnosis and subsequent management of pulp pathology in carious primary molars remain challenging, and clinical studies help optimize treatment. New works are constantly appearing in literature on the description of treatment methods or a comparative assessment of the effectiveness of various materials $[3,4]$. Pulpotomy is a convenient treatment for choosing vital primary exposure to dental pulp, but in the literature among many dentists there is a tendency to have pulpectomy for the treatment of chronic pulpitis of temporary teeth [5].

The purpose of the study is a comparative analysis of pulpectomy (root canal therapy $\mathrm{RCT}$ ) and pulpotomy in temporary molars with chronic fibrous pulpitis.

\section{Materials and Methods}

We aimed to identify patients with carious vital pulp exposure in primary molars if pulpectomy is better than pulpotomy from the point of view of soft tissue pathology, radiographic criteria: pathological root resorption, periapical x-ray transparency, pulp canal

${ }^{*}$ Corresponding author: lb1@mail.ru 
obliteration and tooth survival based on data medical histories. A retrospective study was conducted at the Department of Pediatric Dentistry and Orthodontics, Ural State Medical University. There were examined 198 medical histories of children for the period from 2015 to 2019 with chronic fibrous pulpitis, of which 92 medical histories were selected according to the selection criteria, and panoramic tomograms performed in dynamics were also analyzed.

The inclusion criteria were: children ages 4 to 8 years; diagnosis upon treatment: chronic pulpitis of temporary molars (without pain in the anamnesis); the case of treatment is completed, the child is constantly observed in the clinic; adherence to treatment protocol. The exclusion criteria were: history of systemic disease; chronic pulpitis with pain in the anamnesis; the treatment is incomplete, the child left the clinic; modified treatment protocol.

Treatment protocol: Pulpectomy and pulpotomy with the zinc-oxide eugenol paste (ZOE) has been used. All dental treatment was provided under local anesthesia. Caries removal was obtained with a water-cooled high-speed turbine. Isolation was used. For RCT pulp tissue was removed using endodontic files. The canal was irrigated with chlorhexidine bigluconate $0.05 \%$ solution, dried using a paper pins and obturated with ZOE using a spiral paste filler («Lentulo Spiral Filler», Dentsply). When pulpotomy was used, the coronal pulp tissue was removed with a spoon excavator and irrigated with chlorhexidine bigluconate $0.05 \%$ solution; the hemostasis was obtained with moistened sterile cotton pellets. Zincoxide eugenol paste («Eugetine», Technodent) was directly applied to the radicular pulp. Following the pulp treatment, all teeth were restored («Dyract XP», Dentsply).

The main parameters of the study: age of treatment; post-treatment survival time; tooth loss age; diagnosis during tooth extraction / loss. The main causes of tooth extraction were: second dentition, presence of clinical pathology (swelling, fistula, abscess, positive percussion); presence of radiological failure (radiolucency of the periapical region or furcation, and pathological external root resorption).

All data were analysed using Statistical Package for Social Science (SPSS), version 16. Quantitative data are presented as mean and mean error. To the homogeneity hypothesis of quantitative data, the Student t-test was used, and for the qualitative, the chi-square test was used. Spearman coefficient was used for correlation. The level of significance was set at $\mathrm{p}<0.05$.

\section{Results and Discussion}

Of the 100 completed treatment cases, 50 teeth were treated by pulpectomy, and 50 teeth were treated by RCT, where the first temporary molars are 58 teeth and the second temporary molars are 42 teeth.

Exacerbation of chronic periodontitis (clinical failure) occurred equally often after RCT, and after pulp amputation - it was detected in 8 cases and in 5 cases in each group. No statistically significant differences of clinical failure were found between the groups of treatment. Acute soft tissue reaction may be associated with pathogenic virulence, immunity characteristics and other factors. However, according to our results, the development of clinical failure does not depend on the treatment method. It can be assumed that the smaller the child's age at the time of pulpitis development, the higher the likelihood of complications. In this study we did not find a connection between the initial treatment and clinical failure: pulpitis age and clinical failure did not correlate in both treatment groups ( $\mathrm{rs}=-0.062$, $\mathrm{rs}=-0.103, \mathrm{p}>0.05)$.

$\mathrm{X}$-ray results of completed cases showed failure in 34 cases $(68 \%)$ in the pulpectomy group and in 17 cases (34\%) in the pulpotomy group. Data from other similar studies are better. Radiographical failure in incisors was $41 \%$ in the pulpotomy group and $18 \%$ in 
pulpectomy group at 2 years follow up trial by M.Casas.[6]. Cumulative radiographical results showed failure in $11 \%$ in the pulpotomy group, and $27 \%$ in pulpectomy group in the B.Howley et al. [5]. Our results showed statistically significant difference in radiological failure of pulpectomy compared with pulpotomy (RR 2.0, 95\% CI 1.30 to 3.08). The relative risk of developing periodontitis was twice as high in the root canal treatment group (Table 1.). Our results are consistent with the results of the meta-analysis for radiographic failure, presented by L.Gadallah at al. [4]. The sensitivity analysis for radiographic failure showed RR 0.45 , 95\% CI 0.25 to 0.83 with statistical significant difference between pulpotomy and pulpectomy and a higher risk for radiographic failure in pulpectomy. Pearson Conjugation Ratio also shows that the connection between RCT and radiological failure is relatively strong $\left(\mathrm{C}^{\prime}=0.455\right)$.

Table 1. Comparison of the complications risk after pulpectomy versus pulpotomy in primary molars

\begin{tabular}{|c|c|c|}
\hline & Radiological failure & Clinical failure \\
\hline $\begin{array}{c}\text { Absolute risk in the pulpectomy group } \\
\text { (EER) }\end{array}$ & 0.680 & 0.160 \\
\hline $\begin{array}{c}\text { Absolute risk in the in the pulpotomy group } \\
\text { (CER) }\end{array}$ & 0.340 & 0.100 \\
\hline Relative Risk (RR) & 2.000 & 1.600 \\
\hline Confidence Interval (95\% CI) min & 1.300 & 0.562 \\
\hline Confidence Interval (95\% CI) max & 3.076 & 4.556 \\
\hline p (Chi-square test) & 0.001 & 0.373 \\
\hline Pearson Conjugation Ratio (C') & 0.455 & 0.126 \\
\hline
\end{tabular}

We expected that the method of RCT was more successful than pulpotomy, but did not find any advantages. The stage of tooth development is important when choosing a treatment method. If the period of tooth regression has begun, pulp extirpation can accelerate root resorption. Another important point to raise regarding the fact that pulpotomy histologically preserves the barrier of vital pulp against infection. Many negative diagnostic factors affect the outcome of the treatment: inaccurate history data collected from the parents, subjective perception by the child of diagnostic procedures, interpretation errors of radiographs, impossibility of evaluating pulp infection.

The technical stages of childrens endodontic treatment also have certain difficulties - for example, lack of isolation, difficulties in determining the working length and good canals obturation. The material for root pulp capping, filling the roots is also important. ZOE paste is one of the most commonly used materials, however there are different research results. The total success rates in ZOE pulpotomy groups were only $68 \%$ after 24 months of observation [7]. But R.Najjar,s (2019) meta-analysis shows that ZOE or ZOE/iodoform is the materials of choice for pulpectomy in primary teeth need long time before exfoliation [8].

Post-treatment survival assessment may not be appropriate given the physiological changes in temporary teeth. However, it is reasonable to consider the final result of treatment (Table 2). The post-treatment survival in RCT-group was $26.46 \pm 1.36$ months, the median was 25.00 , and in pulpotomy group was $25.76 \pm 1.47$ months, the median was $27.50(p>0.05)$. Survival time is important for planning the restoration of a tooth after pulp treatment. J.-W. Chen at al.(2020) reports that the median (interquartile range) number of years to relapse teeth with pulpitis after RCT was 3.5 (3.4-3.8) years.

The success of endodontic treatment also depends on appropriate restorative procedures. The results of a study on 1010 endodontically treated permanent teeth demonstrated that the quality of the coronal restoration was significantly more important than the quality of the endodontic filling, considering the apical periodontal health as the outcome [10]. We also 
showed the same time of post-treatment survival (about 2.2 years) with different pulp therapies.

Table 2. Parameters of all molars treatment: age, post-treatment survival

\begin{tabular}{|c|c|c|c|c|c|c|}
\hline treatment & \multicolumn{2}{|c|}{$\begin{array}{c}\text { age of treatment } \\
\text { months/ years }\end{array}$} & \multicolumn{2}{c|}{$\begin{array}{c}\text { age of loss } \\
\text { months/ years }\end{array}$} & \multicolumn{2}{c|}{$\begin{array}{c}\text { post-treatment survival } \\
\text { months/ years }\end{array}$} \\
\hline pulpectomy & $79.52 \pm 2.04$ & 6.6 & $106.56 \pm 1.95$ & 8.9 & $26.46 \pm 1.36$ & 2.2 \\
\hline pulpotomy & $82.60 \pm 2.31$ & 6.9 & $107.78 \pm 2.46$ & 9.0 & $25.76 \pm 1.47$ & 2.2 \\
\hline $\mathrm{p}$ & 0.326 & & 0.701 & & 0.730 & \\
\hline
\end{tabular}

In the present study, no differences of survival were found between the first molar and the second molar (Table 3$)$. The age of the first molar lesion is 6.3 years $(75.93 \pm 1.88)$, the loss is 8.4 years $(100.91 \pm 1.79)$. The age of the second molar lesion is 7.3 years $(88.14 \pm$ $2.20)$, the loss is 9.6 years $(115.79 \pm 2.19)$.

Table 3. Parameters of first molar (M1), second molar (M2) treatment: age, post-treatment survival

\begin{tabular}{|c|c|c|c|c|c|c|c|}
\hline \multirow{2}{*}{ Parameters } & & \multicolumn{2}{|c|}{$\begin{array}{c}\text { age of treatment } \\
\text { months/ years }\end{array}$} & \multicolumn{2}{c|}{$\begin{array}{c}\text { age of loss } \\
\text { months/ years }\end{array}$} & \multicolumn{2}{c|}{$\begin{array}{c}\text { post-treatment survival } \\
\text { months/ years }\end{array}$} \\
\hline \multirow{3}{*}{ pulpecotomy } & M1 & $75.58 \pm 2.10$ & 6.3 & $101.94 \pm 2.03$ & 8.5 & $25.48 \pm 1.60$ & 2.1 \\
\cline { 2 - 9 } & $\mathrm{M} 2$ & $87.17 \pm 3.78$ & 7.3 & $115.53 \pm 3.17$ & 9.6 & $28.35 \pm 2.43$ & 2.4 \\
\cline { 2 - 9 } & $\mathrm{p}$ & 0.006 & & 0.000 & & 0.730 & \\
\hline \multirow{3}{*}{ pulpotomy } & $\mathrm{M} 1$ & $76.40 \pm 3.37$ & 6.4 & $99.60 \pm 3.14$ & 8.3 & $23.16 \pm 1.92$ & 1.9 \\
\cline { 2 - 9 } & $\mathrm{M} 2$ & $88.80 \pm 2.64$ & 7.4 & $115.96 \pm 2.98$ & 9.7 & $28.36 \pm 2.11$ & 2.4 \\
\cline { 2 - 8 } & $\mathrm{p}$ & 0.007 & & 0.000 & & 0.080 & \\
\hline
\end{tabular}

Although the pulpitis is earlier in the first molar, it is later in the second molar - the difference is one year. The frequent and early susceptibility of the first temporary molars in comparison with the second molars depends on the structural features and early eruption.

\section{Conclusion}

This study showed that there is a significant difference in clinical success rates of pulpotomy and pulpectomy in the treatment of carious vital pulp exposure in primary molars. Significant differences in radiological failure rates were found among the groups of pulpectomy and pulpotomy. Since the level of complications after RCT remains high, it is preferable to use pulpotomy in primary molars.

\section{References}

1. P.A.Voronin, T.P.Pliukhina, M.A.Kovalchuk, Pediatric dentistry and dental prophylaxis, 1, 53 (2019)

2. Guidline on pulp therapy for primary and immature permanent teeth. American Academy of Pediatric Dentistry (2014)

3. V. Smaïl-Faugeron, F. Courson, P. Durieux, M. Muller-Bolla, Cochrane Database of Systematic Reviews, 5 (2018)

4. L.Gadallah, M.Hamdy, A.El Bardissy, M.Abou El Yazeed, F1000Res.,7, 1560 (2019)

5. B.Howley, N.S.Seale, A.G.McWhorter et al., Pediatr Dent., 34(5), 112 (2012)

6. M.J. Casas, D.J.Kenny,D.H.Johnston, et al., J Can Dent Assoc., 70(1), 34(2004).

7. T.D. Nguyen, P.L.Judd, E.J.Barrett et al., Pediatr Dent., 39(1), 34 (2017) 
8. R.S.Najjar, N.M.Alamoudi, A.A.El-Housseiny, A.A. Al Tuwirqi, H.J.Sabbagh, Clin Exp Dent Res., 5, 294 (2019)

9. H.Y.Chen H.Li , M. Li , L. Y. Qiyin, S. K. Chen, Int. Pediatr Dent., 30, 225 (2020)

10. H.A.Ray, M.Trope, Int Endod J, 28, 12 (1995) 